\title{
Bibliography of Parts Three and Four
}

Bibliographie des $3^{\text {eme }}$ et $4^{\text {ème }}$ parties Literatur zum dritten und vierten Teil

Symposium sur les urétrites non-gonococciques Monaco, 1954, vol. 1, p. 101 (Masson, Paris 1957)

Symposium international sur les infestations à trichomonas

Reims 1957, vol. 1, p. 381 (Masson, Paris 1958)

Ackermann, A.: Derm Z. 71: 132 (1935).

Allen, E.: Amer. J. Obstet. Gynec. 30: 565 (1935). - Amer. J. Surg. (1936). - Amer.

J. Obstet. Gynec. 45: 246 (1943). - Amer. J. Obstet. Gynec. 51: 387 (1946). Allen, E. and Butler, S.: Amer. J. Obstet. Gynec. 51: 387 (1946). Allison, G. G.: Sth. Amer. J. Med. 36: 82-23 (1943). Appelbaum, E. and Leff, W. A.: J. amer. med. Ass. 138, 119 (1948). Archawsky, M. and Samina, M.: Urologija 13: 255 (1943).

Barnes, J.: Les infestations à trichomonas, p. 352 (1957). - Bull. St. fr. Gynec. 28: 222 (1958). Barnes, J.; Boutwood, A,; Haines, E.; Lewington, W.; Lister, E. and Haram, B. J.:

Brit. med. J. /; 1160-62 (1957). Barretto, M. P.; Filho, Z. M.; Oliveira, P. M. W.; Medonca, J.; Saguis, J.; Gui-

maraes, J. and Vila, E.: Rev. Ass. Med. brazil. 4: 126 (1958). Bauer, H.: Derm. Wschr. 115: 49 (1942). - Z. Urol. 5: 224 (1952). - Zbl. Gynäk. 7: 246

(1952). - Derm. Wschr. 136: 37 (1957). - Rapport Sté de Dermatologie, Erlangen 28 juin (1957). - Gynéc. prat. 8: 361 (1957), - Les Infestations à Trichomonas.

p. 21 (1957). Baum, H. C.: Med. Clin. N. Amer. 42: 263-65 (1958). Bedoya, J. M.: Gynéc. prat. 8: 403 (1957). - Gynéc. prat. 8: 413 (1957). - Tricomonasis

sexual humana. (Facta, Valencia 1959). Bedoya, J. M. and Fdez.Ortega, J. M.: Gynéc. prat. 2: 97 (1958). Bedoya, J. M. and Rios, G.: Geburtsh. Frauenheilk. 10: 989 (1958). Bedoya, J. M., Rios, G. and Rico, L. R.: Geburtsh. Frauenheilk. 8: 990, 994, 997 (1958). Bensen: Arch. Schiffs- u. Tropenhyg. 12: 661 (1908). Beric, B. and Miletic, M.: Zborn. Rad. Inst. Fiziol. (Beograd). Trecieg Kongr. Ginek. Jugoslav. (1956). Bieren, S.: Med. Ann. D. C. 22: 17-22 (1953). Bland, P. B. et al: Amer. J. Obst. Gynec. 32: 835 (1936). - J. amer. med. Ass. 115:

1013 (1940). Bland, P. B. and Rakoff, A. E.: Vida nueva 37: 467 (1936). Bonestell: J. Parasit. 22: 511 (1936). Bordo: West. Vener. Dermat. 3: 54 (1957).

Bourne, A.: British Obstr. and Gynec. Practice, Vol. 2. (Heinemann, London 1955). Brady, L. and Ried, R. D.: Amer. Surg. 115: 840 (1942). Breindl: cit. par Sebek in Shornik lek. 43: 1 (1941). Brewer, J. I.; Halpern, B. and Thomas, J.: Amer. J. Obstet. Gynec. 74: 834 (1957). 166

Bibliography of Parts Three and Four

Bushby, S. R. M.; Catterall, R. D. and Williamson, M.: Brit. med. J. /: 78-80 (1955). Buu-Hoi,N. P.;Xuong, N. D. and Zajdela, F.: Bull. Soc. Chim. Fr. //-/2.-1591 (1955). Buxton, L.: Obstet. Gynec, N. Y., Vol. 12, 6: (1958). Bykhowsky and Gouchansky: Ann. Mai. vénér. 30: 92 (1935). Candiani, G. B.: La Trichomoniasi vaginale. p. 172 (Salpietra, Florence 1953). - Rev. 
Obstet. Ginec. Vol. viii, 2: 101 (1953). Carron, L. A.: Quart. Bull. Northw. Univ. med. Sch. 22: 326-29 (1955). Carson, R. B.: Urol. cutan. Rev. 51: 23 (1957). Catterall, R. D.: Les Infestations à Trichomonas. p. 355 (1957). Catterall, R. D. and Nicol, C. S.: Brit. med. J. 2: 29-31 (1957).

Catterall, R. D. and Williamson, M.: Brit. J. vener. Dis. 31: 119-23 (1955). Cavier, R.; Savel, J. and Rucart, G.: Les Infestations à Trichomonas. p. 323 (1957). Chappaz, G.: Biol. vag. vii, 116: 86 (1946). - Bull. Acad. nat. Méd. 1 and 2: 43 (1955). -

Les Infestations à Trichomonas. p. 161 (1957). Chappaz, G. and Chatellier, X.: Vaginites à Trichomonas. p. 134 (Doin, Paris 1951). Coutts, W. E. and Silva-Inzunza, E.: Acta Venerol. 51: 23 (1952). Coutts, W. E. and Vargas-Salazar, R.: Brit. J. vener. Dis. 24:109-20 (1948). - et al.: Rev. Chil. urol. 15: 48 (1952). - et al: Brit. J. vener. Dis. 28: 97 (1952). - et al.: Brit. med. J. 2: 885 (1955). - et al.: Urol. cutan. Rev. 55: 148 (1951). Cuckler, A. G.; Kupferberg, A. H. and Millman, H.: Antibiot. Chemother., N. Y. 5:

540 (1955). Curtis, A. J.: J. amer. med. Ass. 74: 1706 (1920). Cuthbert, E. J. R.: Practitioner 174: 222-24 (1955).

Davis, C. H.: Amer. J. Obstet. Gynec. 18:196 (1924). - J. amer. med. Ass. 157:126 (1955).

Debiasi: Ann. Ostet. Ginec. 61: 281 (1939).

Del Castillo, A; Galli, M.: J. clin. Endocrin. 9: 1362 (1949).

Deming, J. E.: Northw. med. J. 54: 992 (1955).

Depooter, L.: C. R. Soc. franc. Gynéc. 28: 53 (1958).

Deschiens and Kipschidze: cit. par Simic. Symp. de Reims. (1957).

Dobell: Parasitology 26: 531 (1934). - 31:178 (1939). - 34:101 (1942). - 35:134 (1943).

Dock, G.: Med. News (N. Y.) 65: 690 (1894).

Donné, A.: C. R. Acad. Sci. 3: 385 (1836).

Dunlop, E. M. C; Philipp, E. and Watt, J. D.: Brit. J. vener. Dis. 34: 57 (1958).

Durel, P.: Prophyl. Sanit. Soc. 26 Mars: 48-65 (1954). - Bull. Acad. Med. 3 and 4, 65

(1954). - Gynéc. prat. 6 (1957). - Les Infestations à Trichomonas. p. 114 (1957). -

Gynéc. prat. 8: 459 (1957). Durel, P. and Roiron, V.: Gynéc. prat. 8: 381 (1957). Durel, P.,

Roiron, V., Siboulet, A. et Borel, L. S.: C. R. Soc. franc. Gynéc. 19 Janvier

(1959).

Eldes, C. A.: Amer. J. Obstet. Gynec. 43: 1054 (1942). Escomel: Presse méd. 48: 172 (1940).

Ferguson, R. T. and Edgerton, C. S.: Virginia med. Monthly 77: 643 (1950).

Feinberg, J. G. and Whittington, M. J.: J. clin. Path. 10: 327 (1957).

Feo, L. G.: Amer. J. Obstet. Gynec. 65: 1330 (1953).

Feo, L. G. and Fetter, T. R.: J. Urol. 80:12-1A (1958).

Feo, L. G. and Col: Brit. J. vener. Dis. Vol. xxxii. 4: Dec. (1956). - J. Urol. 75: 711-16

(1956). - Amer. J. trop. Med. Hyg. 24: 195 (1944). Filler, J. W.: Int. Rec. Med. 168: 557-59

(1955). Freed, L. F.: S. Afr. med. J. 22: 223-26 (1948).

Bibliography of Parts Three and Four

167

Garcia, R. A.: Rev. méd. Cubana 50: 428 (1939).

Gardner, H. L. and Dukes, C. D.: Amer. J. Obstet. Gynec. 69- 962 (1955)_Qutt

Gynec, N. Y. 8: 591-96 (1956). $\quad$ vjDstet.

Garducki, J. F.; Timmeus, E. K.; Wilson, L. R.: Sodegren, J. G.; Hettinger B R

and P'an, S. Y.: Antibiot. Chemother., N. Y. 5: 490-495 (1953). Gready, T. G.: Mississippi Doct. 33: 213-14 (1956). Greenblatt, R. B.: West. J. Surg. 53: 135 (1945). Greene, R. J.: Antibiot. 
Chemother., N. Y. 2: 119-22 (1952). Greenhill, J. P.: Amer. J. Obstet. Gynec. 16: 870 (1928). J. amer. med. Ass. 96: 1862

(1931). - Year Book of Obstr. Gyn. p. 454 (1942). - Year Book of Obstr. Gyn. pp.

407-410 (1950). Goodall: J. Obstet. Gynaec. brit. Emp. 45: 597-608 (1938). Grimmer: Z. HautGeschlkr. 7: 1-8 (1949). Grimes, W. A.: J. Urol. 76: 83 (1956).

Hammer, J. M.; Degroat, A. and McGregor, J. R.: J. Mich. med. Soc. 55: 888 (1954). Harkness, A. H.: Non gonococcal urethritis. (Livingstone, Edinburgh 1950). Hegner and Ratcliffe: J.

Parasit. 23: 225 (1937). Hess, E.: Klin. Wschr. 44: 1937 (1933). - Klin. Wschr. 14: 240 (1935). Gynek. Obstet.

34: 191 (1936). - J. Egypt, med. Ass. 21: 813 (1938). Hibbert, G. F.: Amer. J. Obstet. Gynec. 25: 465 (1933).

Hoefer, W. H. V.; Bailey, F. A. and Farley, W. W.: Antibiot. med. 4: 31-34 (1957). Hoehne, O.:

Zbl. Gynäk. 40: 4-15, 113-118 (1916). Hundley, J. M.; Diehl, W. R. and Haggott, J. W.: Amer. J. Obstet. Gynec. 60: 977

(1950).

Iger, J. and Kupperman, R. J.: Int. Rec. Med. 168: 723-30 (1955).

Jira, J.: Zbl. Bakt., I. Abt. Orig. 172: 310-329 (1958).

Jirovec, O. and Rodova, H.: Zbl. Bakt., I. Abt. Orig. 148: 338 (1942).

Johnson, G.: J. Parasit. 28: 369-379 (1942).

Johnson, G. and Kupferberg, A. B.: Proc. Soc. exp. Biol., N. Y. 67: 390-92 (1948).

Johnson, G. and Mayne, R.: Amer. J. Obstet. Gynec. 55: 852 (1948).

Karnaky, K. J.: Sth. med. J. 29: 939 (1936). - Amer. J. Surg. 48: 216 (1940). - West.

J. Surg. 54: 61 (1946). - Amer. J. Obstet. Gynec. 61: 229 (1951). - Postgrad. Med.

9: 220 (1951). - Sth. med. J. 51: 925 (1958). Katsunuma: Bull. Soc. Path. exot. 17: 216 (1924).

Kean, B. H.: Amer. Obstet. Gynec. 70 : 397 (1955). Kean, B. H. and Day, R.: Amer. J. Obstet.

Gynec. 4: 155 (1954). Kean, B. H. and Weld, J.: Proc. Soc. exp. Biol., N. Y. $89: 218$ (1955).

Keutel, H. J.: Gynéc. prat. 8: 423 (1957). - Les Infestations à Trichomonas. p. 151

(1957). - Zbl. Bakt., I. Abt. Orig. 172: 310 (1958). Keutel, H. J. and Neumann: Zbl. Gynäk. 34: 1352 (1955). Kessel and Thomson: Sthw. Med. J. 22: 133 (1938).

Kessel; Thomson and Wilsar: Proc. Soc. exp. Biol., N. Y. 74: 755 (1950). King, A. J. and

Gallagher, E.: Lancet /: 916 (1946). King, A. J. and Marsall: cit. Poizner.

King, A. J.; Mascall, N. and Price, I. N. O.: Lancet 2: 18 (1936). Kiss ling: Gynéc. Obstet. 29: 116 (1934).

Kistner, R. W. and Duncan, C. J.: Obstet. Gynec, N. Y. 4: 155 (1954). Kostic, P.: S. A. 9: 648

(1949). - S. A. 10-11: 751 (1949). - S. A. 4: 354 (1953). - S. A.

/: 19 (1954). - Zborn. Rad. Inst. Fiziol. (Beograd). II. Kongr. akus. gin. Jug. 1954.

ii, 1 (1954). - Arch. Gynäk. 184: 410 (1957).

168

Bibliography of Parts Three and Four

Kreig, E. M. G.: J. Mich. med. Soc. 53: 749 (1954).

Kupferberg, A. B.; Johnson, G. and Sprince, H.: Proc. Soc. exp. Biol., N. Y. 67:

304 (1948). Kupferberg, A. B.; Singher, H. O.; Lampson, G.; Levy, L. and Romano, A. H.:

Ann. N. Y. Acad. Sci. 56: 1006 (1953). Künstler, J.: J. Mickrographie 8: 317 (1884).

Lambillon, J.; Kang, T. and Pelepete, N.: Ann. Soc. beige. Méd. trop. 84:183 (1954)

Lanceley, F.: Brit. J. vener. Dis. 29: 213-17 (1953).

Lanceley, F. and McEntegart, M. G.: Lancet /: 668 (1953). 
Lang, W. R.; Israel, S. L. and Fritz, M. A.: Obstet. Gynec, N. Y. //.- 352 (1958).

Lee, A. F. R. and Keifer, W. S.: Northw. Med. (Seattle). 53: 1227-30 (1954).

Leopold, S.: U. S. armed Forces med. J. 4: 263 (1953).

Lewis, B. and Carole, G.: J. Urol. 19: 337 (1928).

Liston, W. G. and Liston, W. A.: J. Obstet. Gynaec. brit. Emp. 46: 477 (1939).

Loizaga: Bol. Inst. Clin. quir. (B.-Aires) 4: 295 (1928).

Lynch, J.; Holley, E. C. and Margison, J.: Antibiot. Chemother., N. Y. 9: Sept. (1955).

Magara, M.: Gynéc. prat. 6: (1957).

Magara, M.; Amino, E. and Yokouchi, E.: Amer. J. trop. Med. Hyg. Vol. 8. 2: March

(1953). Magara, M.; Nittono, H. and Senda, T.: Antibiot. Med. Vol. 1, 7: (1955). Magara, M.;

Yokouti, E.; Senda, T. and Amino, E.: Antibiot. Chemother., N. Y. 4:

483 (1954). Mallin, M. L. and Seeley, H. W.: Arch. Biochem. 73: 306-14 (1958). McCullagh, W.

M. H.: C. R. Soc. franc. Gynéc. /: 50 (1958). - Lancet /: 698 (1953). McVay, L. V.; Laird, R. L.;

Flanagan, J. R. and Sprtjnt, D. H.: Proc. Soc. exp. Biol.,

N. Y. 72: 674-5 (1949). Moore, S. F, Jr. and Simpson, J. W.: Amer. J. Obstet. Gynec. 68: 974 (1954). Morgan : J. Parasit. Suppl. 27:16 (1941). - J. Parasit. 31:8 (1949). - Bovine Trichomoniasis. (Burgess, Minneapolis 1944).

Ottolenghi-Preti, G. F.: Les infestations à trichomonas. p. 129 (1957).

Nicol, C. S.: Brit. J. vener. Dis. 34: 192 (1958).

Nicol, C. S.; Gallagher, E. and King, A. J.: Brit. J. vener. Dis. 28: 142-3 (1952).

Ninomiya, H. and Suzuoki, Z.: J. Biochem. (Tokyo) 39: 321-331 (1952).

Pace, H. R. and Schantz, S. L.: J. amer. med. Ass. 162: 268 (1956).

Pattyson, R. A.: N. Y. St. J. Med. 37: 41 (1937).

Perez, M. L. and Blanchard, O.: Tricomoniasis vaginal, p. 173. (El Alteneo, Buenos-Aires 1944.)

Perl, G.; Guttmacher, A. F. and Raggazoni, H.: Obstet. Gynec, N. Y. 7:128 (1956).

Perin; Lissmann and Senghor: Rapport Journées Dermat. p. 364-67. Lyon, 26-27 mai, 1951.

Pestre: These (Alger. 1953).

Peter, R.: Prometheus-Verlag. p. 136, Prague 1945. - Les Infestations à Trichomonas. p. 155

(1957). - Gynéc. prat. 6: 431-44 (1957).

Peter, R. and Jirovec, O.: Prometheus-Verlag. p. 135, Prague 1945. - Proph. 1:102-285 (1954).

Plentl, A. A.; Gray, M. J.; Nelsen, E. D. and Dalali, S. J.: Amer. J. Obstet. Gynec. 71: 116

(1958).

Poizner and Koulakowa : Edit, a Tomak. - Trad. Centre Econ. et Cult. France-U.R.S.S. (1952).

Priddle, H. D. and Gar Li, G.: Amer. J. Obstet. Gynec 65: 166-9 (1953).

Bibliography of Parts Three and Four

169

Raddin, J. B.: Clin. Med. 4: 1111-13 (1957).

Rakoff, A. E.: Amer. Obstet. Gynec. 42: 267-80 (1947).

Ray, J. L. and Maughan, G. M.: West. J. Surg. 64: 581 (1956).

Read, C. P. and Rothman, A.: Amer. J. Hyg. 61: 249-260 (1955).

Reich, W. J.; Nechtow, M. J.; Rubinstein, H. W. and Doswald, A. M.: Illinois med.

j. 108: 278-9 (1955). Riba, L. W.: Arch, surg., Chicago 73: 833-58 (1956). Riff: Gynéc. Obstét. p. 274 (1934).

Rodecurt, M.: Dtsch. med. Wschr. 45: 546 (1933). - Zbl. Gynäk. 60: 567 (1936). Rogers, C. S.; Bellott, C. B.; Paul, M. F.; Yurchenco, J. A. and Gever, G.: Antibiot. 
Chemother., N. Y. 6: 231-42 (1956). Roland, M.; Yeprovsky, E. C. and Lenhart, W.: Obstet. Gynec, N. Y. 7: 459-61

(1956). Roth, R. B.: J. vener. Dis. Inform. 25: 163-66 (1944).

Salisbury, J. H.: Amer. med. Sci. 55: 371 (1868).

Sampaio: Trab. Soc. port. Derm. Vener. 13: 255 (1955).

Don Santos and Zagury: An. bras. Ginec. 16: 157 (1943).

Schaw-Henriksen and Kessel: West. J. Surg. 60: 563 (1952).

Schmidt and Kamniker: Arch. Gynäk. 127: 362 (1926).

Schroeder, R.: Zbl. Gynäk. 45: 410 (1957).

Seneca and Ides: Amer. J. trop. Med. Hyg. 2: 1948 (1953).

Siboulet, A.: J. Méd. Chir. prat. 9: 399-403 (1959).

Simic, C.: Les Infestations à Trichomonas. pp. 233-36 (1957). - Protoz. ii: 77 (1940).

Simic, C. and Petrovic Z.: Gl. SSV. San. knj. (1952).

Sorel, C: J. Urol. 58: 109-17 (1952).

Stabler, R. M.; Feo, L. G. and Rakoff, A. E.: J. Parasit. 26: Suppl. 22 (1940). - Amer.

J. Hyg. 42: 276 (1941). - Amer. J. Hyg. 45: 529 (1943). Stein, I. F.: quoted by Trussel, R. E. (1943). Stein, I. F. and Cope, E. J.: Amer. J. Obstet. Gynec. 22: 368 (1931). - Amer. J. Obstet. Gynec. 25: 819 (1933). Stewart, H. C; Hughes, W. R.; Thomas, E. G. and Nixon, W. C. W.:

Lancet 2:1028

(1957). Strain, R. E.: J. Urol. 54: 483 (1945). Swartzwelder, J. C; Mule, J. C; Frye, W. W. and Vella, F.: Med. Times 83: 704

(1955). Symposium sur la Trichomonase; International Record of Medicine . General Practic Clinics, Sept. a. Nov. 1955, Vol. 168, No. 9 a. No. 11. pp. 551-565, 709-730.

Teocharow: Vestn. Vener. Derm. 30: A $>$ >-1 (1956).

Thiery, M.: Les Infestations à Trichomonas. p. 370 (1957). - Bull. Soc. franc. Gynéc. 28: 223 (1958). Tinsley, W. H.: Tex. St. J. Med. 51: 5-7 (1954). Trussell, R. E.: J. Iowa St. med. Soc. 30:66 (1940). - Proc. Soc. exp. BioL, N. Y. 47:176

(1941). - J. infect. Dis. 69: 18 (1941). - Amer. J. Obstet. Gynec. 44: 292 (1942). -

J. Parasit. 32: 573 (1946). - Trichomonas Vaginalis and Trichomoniasis. p. 103.

(Blackwell, Oxford 1947). Trussell, R. E.; Wilson, M. E,; Longwell, S. H. and Langhlin, K. A.:

Amer. J.

Obstet. Gynec. 44: 292 (1942).

Wagner, O. and Hess, E.: Zbl. Bakt., I. Abt. Orig. 135: 310 (1935). - Zbl. Bakt., I. Abt. Orig. 138: 273 (1937). Waletsky, E.: Turkey world. May 1950.

170

Bibliography of Parts Three and Four

Walker, R. I.; Goldberger, M. A. and Lapid, M. S.: N. Y. St. J. Med. 51: 937 (1951).

Weiler: Z. Hyg. InfektKr. 121: 27 (1938).

Wellerson, R. and Kupferberg, A. B.: Publication pending (1959).

Wessels, M.: quoted by Trussell, R. E. (1943).

Westphal: Zbl. Bakt. I. Abt. Orig. 137: 363-76 (1936).

Whittington, M. J.: J. Obstet. Gynaec. brit. Emp. 58: 398-405 (1951). - Brit. J. vener. Dis. 33: 80 (1957). - Bull. Inst. Techn. Vener. 4: 61-67 (1958). Wilkins, J. R. and Henshaw, C. T.: Exp. Parasit. 31: 143-54 (1954). Willcox, R. R.: Brit. J. vener. Dis. 33: 115-17 (1957). - Acta derm.-vener. 37: 327-31

(1957). - Brit. J. vener. Dis. 35: 35-37 (1959). - J. Obstet. Gynaec. brit. Emp., in 
press (1959). Wille: Med. Klin. /.- 520 (1918).

Wirtschafter, S. K. and Jahn, T. L.: J. Protozool. 3: 83-85 (1956). Wirtschafter, S. K.; Saltman, P. and Jahn, T. L.: J. Protozool. 3: 86-88 (1956). Woodall, P. S.; Waldro, E. C. and Winkler, C. B.: Obstet. Gynec, N. Y. 6: 283-4

(1956). Wu Yeng: Zbl. Bakt., I. Abt. Orig. 141: 411 (1938).

Yaneva, H. and James, M.: C. R. Soc. franc. Gynéc. 27: 421 (1958). Yang, Y. C. and Li, T. F.: Chin. J. Obstet. Gynec. 5: 19-20 (1957). Youngblood, V. H.: J. Urol. 70: 926 (1953). Urologists Letter Club, April 15, 1959. Youngblood, V. H.; Tomlin, E. M. and Davis, J. B.: J. Urol. 78: 150 (1957). Youngblood, V. H.; Tomlin, E. M.; Williams, J. O. and Kimmdstiel: J. Urol. 79: 120 (1958).

Zebovitz, E.; Evans, J. B. and Niven, C. F.: J. Bact. 70: 686 (1955). 